\title{
Blind spectrum sensing for cognitive radio over time-variant multipath flat-fading channels
}

\author{
Chenglin Zhao ${ }^{1}$, Mengwei Sun ${ }^{*}$, Bin $\mathrm{Li}^{1}$, Long Zhao ${ }^{1}$ and Xiao Peng ${ }^{2}$
}

\begin{abstract}
Cognitive radio has more extensive application in recent years, and it may operate in complex wireless environmental condition such as communication systems with time-variant multipath flat-fading channel. As an essential technology for cognitive radio, most existing spectrum sensing methods are designed for time-invariant propagation channel; thus, it could be extremely difficult to achieve acceptable sensing performance when we apply them to deal with time-variant multipath fading channel. In order to overcome this obstacle, we design a novel spectrum sensing method in this investigation. Firstly, a dynamic state-space model is proposed in which two different hidden Markov models are employed to abstract the evolution of primary user state and time-variant multipath flat-fading channel gain. Based on the dynamic state-space model, the spectrum sensing problem is formulated as blind estimation problem. Relying on maximum a posteriori probability criterion and particle filtering technology, a joint estimation algorithm of the time-variant channel gain and primary user state is presented. Experimental simulations demonstrate the superior performance of our presented sensing scheme, which could be used potentially in realistic cognitive radio systems.
\end{abstract}

Keywords: Spectrum sensing; Time-variant multipath flat-fading channel; Dynamic state-space model; Joint estimation; Particle filtering

\section{Introduction}

The development of various wireless technologies and an increasing demand for high data rate wireless services result in growing shortage of spectrum resources. However, the statistical report given by the Federal Communication Commission (FCC) indicates that the spectrum utilization in many frequency bands is very low [1]. This phenomenon is caused by the mismatch of conventional static spectrum management and the dynamic way radio resources are used. As a result, the contradiction between scarce frequency resources and under-utilization of spectrum restricts the development of wireless communication seriously. Cognitive radio (CR) technique is a promising method to improve the efficiency of spectrum utilization significantly by allowing secondary users (SUs) to utilize the allocated frequency bands when these bands are detected inactive [2-5]. In order to take advantage of $\mathrm{CR}$, a number of wireless standards have developed

\footnotetext{
* Correspondence: mwsun@bupt.edu.cn

${ }^{1}$ Beijing University of Posts and Telecommunications (BUPT), Haidian, Beijing 100876, China

Full list of author information is available at the end of the article
}

including cognitive features, such as IEEE 802.22 [6], IEEE $802.11 \mathrm{k}$ [7], and Bluetooth [8]. IEEE 802.22 standard is known as a cognitive radio standard which contains cognitive features and spectrum sensing requirements.

In order to ensure the quality of service (QoS) of primary users (PUs), the SUs should detect the allocated spectrum frequently, the reasons involve two aspects: firstly, if the allocated spectrum is detected inactive, the SUs could utilize it for communication; secondly, whenever the PUs become active, the SUs must vacate the channel immediately. For reasons mentioned above, spectrum sensing is a fundamental and critical element of $\mathrm{CR}$, and the main propose of spectrum sensing is to detect spectrum accurately and determine whether it is available for SUs at a particular place and time [9]. There have been several classical methods of spectrum sensing. The most common methods are energy detection (ED) $[10,11]$, matched filtering detection (MFD) [12,13], cyclostationary feature detection [14], and waveform-based sensing [15]. Among these methods, MFD could achieve the highest sensing accuracy in the shortest sensing time [16]. Given that pilot, synchronization code, and spread spectrum code are utilized in quantities 
of wireless communication systems, MFD has a wide range of application. However, MFD is a parametric hypothesis method from the statistic view, so the sensing performance is susceptible to the parameters' value setup; the determination of parameters' value is a much more formidable undertaking, and the accuracy of MFD may degrade significantly under time-variant multipath flat-fading (TVMFF) channels which are common in the next-generation communication systems. It is worthy to note that CR technology is expected to be utilized in the next-generation communication systems such as mobile communication and indoor communication, and there is no effective spectrum sensing method to deal with TVMFF channels.

In this paper, we propose a new method which could greatly improve sensing performance over TVMFF channel. The state of PU is decided by detecting whether pilot, synchronization code, or spread spectrum code exists. The innovation of our proposed algorithm involves two aspects: firstly, a novel dynamic state-space model (DSM) is designed to describe aptly the actual spectrum sensing process over TVMFF channel based on matched filtering (MF). In this model, a two-state Markov chain is employed to characterize the evolution of PU state. Given that the transmitted signal of PU and channel amplitude are independent of each other, we adopt data-independent finite state Markov channel (FSMC) to model the TVMFF channel [17]. On these bases, by referring to the traditional MPD sensing scheme, the received signal processed by MF is viewed as the observation for SU; thus, the proposed algorithm could inherit the advantages of MFD such as rapidity and accuracy. Secondly, based on the DSM established, we propose a promising algorithm which could estimate the TVMFF channel gain and the PU state jointly and in real time. Compared with traditional sensing methods which are overwhelmed by time-variant channels and covariance absolute value (CAV) detection algorithm which is newly introduced and has found a wide application recently [18], the originality of our algorithm is that it could estimate the fading channel gain accurately; thus, the sensing performance based on the result of channel estimation could be improved significantly, and the SU could gain insights into surrounding environmental conditions.

The rest of this paper is organized as follows. Section 2 provides the DSM of spectrum sensing over TVMFF channel. In Section 3, we present a simple review of maximum a posteriori probability (MAP) criterion and particle filtering [19]. On this foundation, the joint blind estimation algorithm of PU state and fading channel gain is introduced in detail in Section 4. Numerical simulations and performance analysis are provided in Section 5. Finally, conclusion is generalized in Section 6.

\section{System model}

We consider a spectrum sensing communication system, in which the transmitted signal of PU is contaminated by a TVMFF channel with additive Gaussian white noise. The block diagram of this system is shown in Figure 1. Given that the state of PU is established by detecting whether the pilot of PU signal exists, the $\mathbf{x}_{n}$ represents the pilot sequence at the $n$th sensing slot. The TVMFF channel impulse response is denoted by $\mathbf{h}_{n} \cdot \mathbf{v}_{n}$ represents additive Gaussian white noise, and it is a sequence consisting of zero-mean independent and identically distributed (i.i.d.) random variables, i.e., $\mathbf{v}_{n} \sim N\left(\mathbf{0}, \sigma^{2} \mathbf{I}\right)$. At the $\mathrm{PU}$ receiver end, the received signal is processed by MF and then we can get the observation denoted by $y_{n}$.

Because both the PU state and channel gain have a time-variant characteristic, the DSM we designed gives full consideration to the evolution of these two states and could be represented as follows:

$$
\begin{aligned}
& S_{\mathbf{x}_{n}}=\Gamma\left(S_{\mathbf{x}_{n-1}}\right) \\
& \mathbf{h}_{n}=\Phi\left(\mathbf{h}_{n-1}\right) \\
& y_{n}=\Psi\left(\mathbf{h}_{n}, \mathbf{x}_{n}, \mathbf{v}_{n}\right)
\end{aligned}
$$

The state of PU $S \mathbf{x}_{n}$ and the TVMFF channel gain $\mathbf{h}_{n}$ are hidden to SU, and they evolve according to the state equations $\Gamma($.$) and \Phi($.$) , respectively, Based on the observation$ equation $\Psi($.$) , the observation y_{n}$ is related to the channel

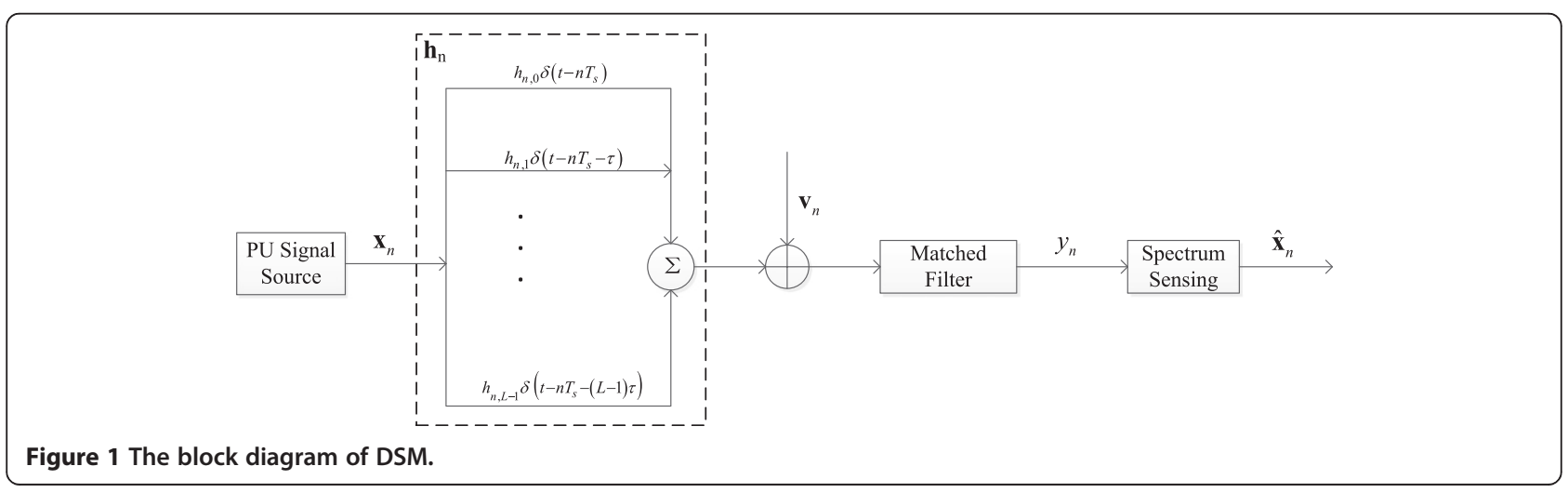


amplitude, sampling value of transmitted pilot, and noise level. We will describe these three equations in more detail in the following sections.

In this paper, we set the length of the pilot as $M$ and the total number of multipath as $L$. For the sake of convenience, the sampling size in one sensing slot equals to the length of pilot and the sampling point is supposed to keep accordance with the change of pilot elements. Furthermore, the PU state is assumed unchanged in a sensing slot. The period of sensing slot is set to be $T_{\mathrm{s}}$, the sampling period is illustrated by $\tau$, and the channel coherent time is denoted by $T_{\mathrm{c}}$. There are quantitative relationships among the aforementioned variables, i.e., $T_{\mathrm{s}}=M \times \tau, T_{\mathrm{c}}=T_{\mathrm{s}} / f_{\mathrm{d}}$. Here, $f_{\mathrm{d}}$ represents the Doppler frequency.

\subsection{PU states}

The working state of PU comes in two forms: inactive and active. We utilize $S_{0}$ and $S_{1}$ to represent them, respectively. They transfer to each other with specified probability, as shown in Figure 2. In this figure $p_{01}=1-$ $p_{00}$ and $p_{10}=1-p_{11}$. The configurations of the transition probability depend on practical application.

There are two hypotheses: (1) $H_{0}$ denotes that the pilot signal does not exist, i.e., the PU is inactive. (2) $H_{1}$ represents that the pilot signal exists, i.e., the PU is active $[16,20]$. We utilize $\mathbf{s}_{\mathrm{c}}$ to denote the pilot, and the transmit signal in $n$th sensing slot could be written as

$$
\mathbf{x}_{n}= \begin{cases}\mathbf{0} & H_{0} \\ \mathbf{s}_{\mathrm{c}} & H_{1}\end{cases}
$$

\subsection{TVMFF channel model}

Time-variant multipath flat-fading channels with memory are very common in wireless communication systems, especially in mobile radio communication systems [21]. This paper's goal is to provide an efficient method to alleviate the noticeable decline in sensing performance caused by TVMFF channel; thus; adopting an accurate channel model becomes absolutely critical. In this paper, the multipath channel is modeled as a linear filter with a time-variant impulse response, and different channels are independent with each other. Furthermore, we utilize the first-order FSMC to model every single channel which is regarded as a finite-state Markov chain. The first-order FSMC model

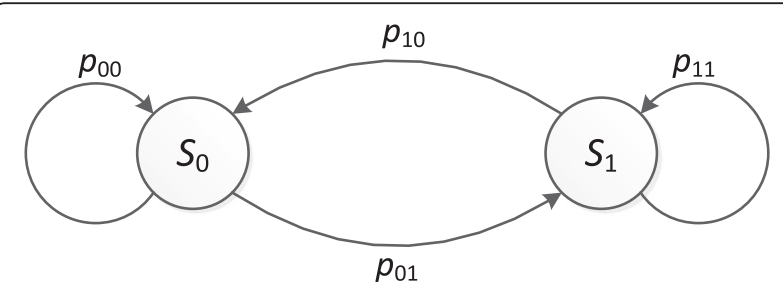

Figure 2 The two-state Markov transition model of the PU state. means that the current channel state is not associated with previous states or future states but the right prior state. In the following part, we will firstly propose the TVMFF channel model and then introduce how to utilize FSMC to model every single channel.

\subsubsection{TVMFF channel model}

As the other hidden state, the impulse response of timevariant multipath flat-fading channel at the $n$th sensing slot could be written as

$$
\mathbf{H}_{n}=\sum_{l=0}^{L-1} h_{l, n} \delta(t-n T s-l \tau) \quad n=0,1, \ldots, N-1
$$

As mentioned above, $L$ represents the total number of multipath channel components. Based on the reference of excess delay bins which means discretizing the multipath delay axis into equal time delay segments, we set the delay time of the $l$ th channel to be $l \tau$. What needs to be stressed is that the delay time of every channel is fixed and time-invariant but the amplitude is timevariant. We use $\mathbf{h}_{n}$ to denote a vector contained by amplitudes of all channels at the $n$th sensing slot, and it could be showed as $\mathbf{h}_{n}=\left[h_{0, n}, h_{1, n}, \ldots, h_{L}-1, n\right]^{T}$. Here, [.] ${ }^{T}$ stands for the transpose of a matrix or vector.

It is worthy to note that the fading channel considered is set to be slow fading. It means that the channel impulse response changes much slower than the period of sensing slot, i.e., $T_{\mathrm{c}}>>\mathrm{T}_{\mathrm{s}}$. In other words, the channel gain is assumed to be unchanged over several bandwidth intervals. For simplicity, $T_{\mathrm{c}}$ is supposed to be multiples of $T_{\mathrm{s}}$, i.e., $T_{\mathrm{c}}=J T_{\mathrm{s}}$, where $J$ is an integer which is much larger than 1. Therefore, due to the different location in a channel gain period, the sensing slots could be classified into two categories: first slot and non-first slot, and they are defined as (6). The frame structure is shown in Figure 3. It is obvious that the channel gain is possible to transfer into another state only in the first slot.

$$
\bmod \left(n T_{\mathrm{s}}, T_{\mathrm{c}}\right)\left\{\begin{array}{l}
=0, \quad \text { First slot } \\
\neq 0,
\end{array} \text { Non-first slot } \quad n=0, \ldots, N-1\right.
$$

As mentioned above, the TVMFF channel gain and the PU state will be estimated jointly and in real time. To guarantee the accuracy of estimation, the channel gain will be estimated in every sensing slot according to different mechanisms which will be introduced in detail in Section 4.2 and then the PU state could be detected based on the observation and estimation result of channel gain.

\subsubsection{FSMC model of single channel}

Because of its wide versatility and computational simplicity, the FSMC model has found wide application for modeling 


\begin{tabular}{|c|c|c|c|}
\hline \multicolumn{2}{|c|}{ channel coherent time } & \multicolumn{2}{|c|}{ channel coherent time } \\
\hline first slot & non-first slot & first slot & non-first slot \\
\hline $\begin{array}{l}\text { Sensing } \\
\text { slot }\end{array}$ & $\cdots$ & & .. \\
\hline$\leftarrow T_{s} \rightarrow$ & & $\leftarrow T_{s} \rightarrow$ & \\
\hline
\end{tabular}

Figure 3 Frame structure of spectrum sensing.

wireless flat-fading channels $[17,22,23]$. The main idea of data-independent FSMC model is partitioning each channel gain into $K$ different discrete states, and every state has its own corresponding steady-state probability and region boundaries of channel amplitude. Moreover, different states may transfer to each other in specified transition probabilities. We will give the specific measures to calculate the aforementioned parameters in the following.

Firstly, the random fading gain of each channel is set to follow a Weibull distribution with different parameters. The probability distribution function (PDF) of the $l$ th fading channel gain is given by [24]:

$$
f\left(h_{l}\right)=\left\{\begin{array}{ll}
\frac{\beta_{l}}{\lambda_{l}}\left(\frac{h_{l}}{\lambda_{l}}\right)^{\beta_{l}-1} \exp \left(\frac{h_{l} \beta_{l}}{\lambda_{l} \beta_{l}}\right) & h_{l} \geq 0 \\
0 & h_{l}<0
\end{array},\right.
$$

where $h_{l}$ represents the amplitude of the $l$ th channel; $\lambda_{l}>0$ is a scale parameter, and $\beta_{l}>0$ is a shape parameter. It should be noted that $l$ represents the label of a single channel, and $l=0,1, \ldots, L-1$.

We set the steady-state probabilities of all FSMC states as equal, i.e., $\pi_{l . k}=1 / K$, and the region boundaries could be achieved by [25]

$$
v_{l, k}=\sqrt[\beta l]{-\ln \left(1-\frac{(k)}{K}\right)} \times \lambda_{l} \quad k=0,1, \ldots, K-1
$$

The non-overlapping regions of the $l$ th partitioned channel amplitude $h_{l}$ could be denoted by

$$
V_{l}=\left\{\left[v_{l, 0}, v_{l, 1}\right),\left[v_{l, 1}, v_{l, 2}\right), \ldots,\left[v_{l, K-1}, \infty\right)\right\}
$$

The statistical channel amplitude corresponding to the $k$ th state is defined as

$$
h_{l, k}=\frac{\int_{v_{l, k}}^{v_{l, k+1}} h_{l} \cdot f\left(h_{l}\right) d h_{l}}{\int_{v_{l, k}}^{v_{l, k+1}} f\left(h_{l}\right) d h_{l}} \quad k=0, \ldots, K-1
$$

Secondly, we utilize $N_{k}$ to denote the level crossing rate (LCR) which is defined as the number of times per second that the fading amplitude crosses the amplitude boundary threshold $v_{k}$ in a downward direction, and $R_{k}$ represents the average number of symbols that are transmitted in state $k$ per second. The configurations of $N_{k}$ and $R_{k}$ could be calculated by (11) and (12), respectively [25].

$$
\begin{aligned}
& N_{l, k}=\frac{f_{\mathrm{d}}}{\lambda_{l}^{2 \beta_{l}}} v_{l, k}^{\beta_{l}-1} \exp \left[-\left(1+v_{l, k}^{\beta_{l}} / \lambda_{l}^{k}\right)\right] \Gamma\left(\frac{1}{\beta_{l}}\right) \\
& R_{l . k}=\frac{\pi_{l, k}}{T_{\mathrm{s}}}
\end{aligned}
$$

Finally, the transition probability between the channel states is formulated based on the aforementioned parameters. The transition probability between the $l$ th channel states is defined as the probability of transition from state $k$ at time index $n-1$ to state $k^{\prime}$ at time index $n$, and it could be written as

$$
p_{l, k \rightarrow k^{\prime}} \triangleq \operatorname{Pr}\left(S_{h_{l, n}}=k^{\prime} \mid S_{h_{l, n-1}}=k\right) \quad k, k^{\prime}=0,1, \ldots, K-1
$$

To calculate the transition probabilities defined in (13), we make an assumption that each channel state can have no more than three outgoing and incoming transitions as shown in Figure 4 [26]. In other words, the channel state could only stay in the same state or transfer to its immediate neighboring state at next time index, and it could be denoted by

$$
\begin{gathered}
p\left(S_{h_{l, n}}=k^{\prime} \mid S_{h_{l, n-1}}=k\right)= \begin{cases}p_{l, k \rightarrow k^{\prime}} & \left|k^{\prime}-k\right| \leq 1 \\
0 & \left|k^{\prime}-k\right|>1\end{cases} \\
k, k^{\prime}=0, \ldots, K-1
\end{gathered}
$$

The general computational method of transition probabilities is given by (15), (16), and (17) [26]:

$$
\begin{aligned}
& p_{l, k \rightarrow(k-1)} \approx \frac{N_{l, k}}{R_{l, k}} \quad k=1,2, \ldots, K-1 \\
& p_{l, k \rightarrow(k+1)} \approx \frac{N_{l, k+1}}{R_{l, k}} \quad k=0,1, \ldots, K-2 \\
& p_{l, k \rightarrow k}=\left\{\begin{array}{lc}
1-p_{l, k \rightarrow(k-1)}-p_{l, k \rightarrow(k+1)} & k=1,2, \ldots, K-2 \\
1-p_{l, k \rightarrow(k+1)} & k=0 \\
1-p_{l, k \rightarrow(k-1)} & k=K-1
\end{array}\right.
\end{aligned}
$$

In this situation, the transitional probability matrix (TPM) $\mathbf{P}_{l}$ of the $l$ th channel contained by transitional probability between channel states is a matrix whose non-zero elements only occur in principal diagonal and 
the two diagonal lines next to the principal diagonal, and it could be represented as follows:

$$
\mathbf{P}_{l}=\left[\begin{array}{ccccccc}
p_{l, 0 \rightarrow 0} & p_{l, 0 \rightarrow 1} & 0 & \ldots & 0 & 0 & 0 \\
p_{l, 1 \rightarrow 0} & p_{l, 1 \rightarrow 1} & p_{l, 1 \rightarrow 2} & \ldots & 0 & 0 & 0 \\
0 & p_{l, 2 \rightarrow 1} & p_{l, 2 \rightarrow 2} & \ldots & 0 & 0 & 0 \\
\vdots & \vdots & \vdots & \ddots & \vdots & \vdots & \vdots \\
0 & 0 & 0 & \cdots & p_{l,(K-3) \rightarrow(K-3)} & p_{l,(K-3) \rightarrow(K-2)} & 0 \\
0 & 0 & 0 & \ldots & p_{l,(K-2) \rightarrow(K-3)} & p_{l,(K-2) \rightarrow(K-2)} & p_{l,(K-2) \rightarrow(K-1)} \\
0 & 0 & 0 & \cdots & 0 & p_{l,(K-1) \rightarrow(K-2)} & p_{l,(K-1) \rightarrow(K-1)}
\end{array}\right]_{K \times K}
$$

Because any channel is independent of each other. The transition probability between channel states is defined by

$$
\begin{aligned}
\mathbf{h}_{n} \sim p\left(\mathbf{h}_{n} \mid \mathbf{h}_{n-1}\right) & =\prod_{l=0}^{L-1} p\left(h_{l, n} \mid h_{l, n-1}\right) \\
=\prod_{l=0}^{L-1} p\left(S_{h_{l, n}}\right. & \left.=k \mid S_{h_{l, n}}=k^{\prime}\right)=\prod_{l=0}^{L-1} p_{l, k \rightarrow k^{\prime}}
\end{aligned}
$$

\subsection{Observation}

The achievement of observation contains three steps: firstly, the pilot sequence of PU transmitted signal is convolved by the multipath channel impulse response. Secondly, the convolution is added by additive Gaussian white noise. Finally, the received signal is processed by MF and then we can get the observation. In summary, this process could be represented by

$$
y_{n}=\left(\mathbf{x}_{n} \otimes \mathbf{H}_{n}+\mathbf{v}_{n}\right) \otimes \mathbf{s}_{\mathrm{c}}=\left(X_{n}^{T} \times \mathbf{h}_{n}+\mathbf{v}_{n}^{T}\right)^{T} \times \mathbf{s}_{\mathrm{c}}
$$

Here, $\mathbf{v}_{n}=\left[v_{n, 0}, v_{n, 1}, \ldots, v_{n, M-1}\right]$ and $v_{n, m}$ represents the random additive noise value with zero mean and variance $\sigma^{2}$, i.e., $v_{n, m} \sim \mathrm{N}\left(0, \sigma^{2}\right) . \boldsymbol{s}_{\mathrm{c}}$ is a column vector whose length is $M$. ' $\otimes$ 'denotes convolution operation. $\mathrm{X}_{n}$ represents a matrix contained by sampled signal, and it could be written as

$$
X_{n}=\left[\begin{array}{ccccc}
x_{n, 0} & x_{n, 1} & \ldots & x_{n, M-2} & x_{n, M-1} \\
x_{n-1, M-1} & x_{n, 0} & \ldots & x_{n, M-3} & x_{n, M-2} \\
\vdots & \vdots & \ddots & \vdots & \vdots \\
x_{n-1, M-L+2} & x_{n-1, M-L+3} & \ldots & x_{n, M-L} & x_{n, M-L+1} \\
x_{n-1, M-L+1} & x_{n-1, M-L+2} & \ldots & x_{n, M-L-1} & x_{n, M-L}
\end{array}\right]_{L \times M}
$$

\section{Sequential MAP estimation and particle filtering}

The fundamental purpose of this investigation is to design an algorithm which could detect the PU state when the channel is time-variant, and its parameters are unknown to the SU. Based on the DSM described in Section 2, we adopt Bayesian inference and PF methodology to derive a blind scheme for MAP data detection that operates sequentially and recursively. In this section, we will make a detailed introduction about the MAP criterion and PF technology.

\subsection{Sequential MAP estimation}

From the Bayesian view point, MAP criterion is an estimation method that chooses an estimation of unknown variable which could maximize the posterior probability distribution function, i.e.,

$$
\hat{\theta}=\arg \max _{\theta} p(\theta \mid y)
$$

Here, $\theta$ is the unknown variable to be estimated, and $y$ denotes the observation. As we all know, $p(\theta \mid y)=p(y \mid \theta) p$

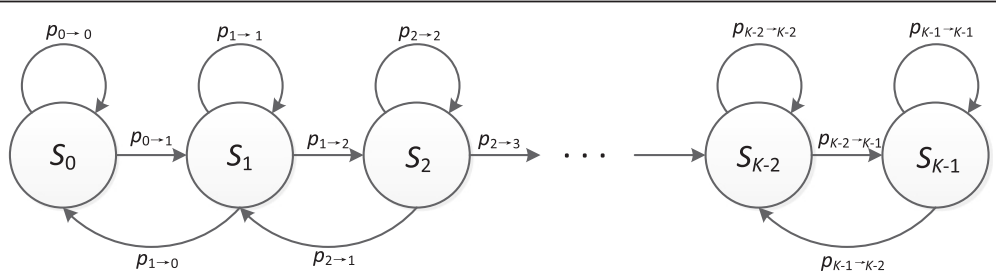

Figure 4 First-order FSMC transition model of the TVFF channel. 
$(\theta) / p(y)$; thus, the MAP estimation of $\theta$ could be represented as

$$
\hat{\theta}=\arg \max _{\theta} \frac{p(y \mid \theta) p(\theta)}{p(y)}=\arg \max _{\theta} p(y \mid \theta) p(\theta)
$$

The aim of this paper is to estimate the TVMFF channel gain and PU state jointly based on the observed symbols. From the Bayesian perspective, the joint estimation could be achieved by MAP criterion, and it could be written as

$$
\begin{aligned}
\left(\hat{\mathbf{h}}_{0: n}, \hat{\mathbf{x}}_{0: n}\right)^{(\mathrm{MAP})} & =\arg \max _{\mathbf{h} \in \mathrm{H}, \mathbf{x} \in \mathbf{X}}\left[p\left(\mathbf{h}_{0: n}, \mathbf{x}_{0: n} \mid y_{0: n}\right)\right] \\
& =\arg \max _{\mathbf{h} \in \mathrm{H}, \mathbf{x} \in \mathbf{X}}\left[p\left(y_{0: n} \mid \mathbf{h}_{0: n}, \mathbf{x}_{0: n}\right) p\left(\mathbf{h}_{0: n}, \mathbf{x}_{0: n}\right)\right]
\end{aligned}
$$

It is obvious from (24) that the MAP estimation leads to extremely high computational complexity. To solve this problem, we can re-write (24) as an iterative and sequential form.

$$
\begin{aligned}
p\left(\mathbf{h}_{0: n}, \mathbf{x}_{0: n} \mid y_{0: n}\right)= & p\left(y_{n} \mid \mathbf{h}_{0: n}, \mathbf{x}_{0: n}, y_{0: n-1}\right) p\left(\mathbf{h}_{n}, \mathbf{x}_{n} \mid \mathbf{h}_{0: n-1}, \mathbf{x}_{0: n-1}\right) \\
& p\left(\mathbf{h}_{0: n-1}, \mathbf{x}_{0: n-1} \mid y_{0: n-1}\right) \\
= & p\left(\mathbf{h}_{0}, \mathbf{x}_{0}\right) p\left(y_{0} \mid \mathbf{h}_{0}, \mathbf{x}_{0}\right) \prod_{n^{\dagger}=1_{n}}^{n} \\
& p\left(y_{n^{\dagger}} \mid \mathbf{h}_{0: n^{\dagger}}, \mathbf{x}_{0: n^{+}}, y_{0: n^{\dagger}-1}\right) \prod_{n^{\dagger}=1} \\
& p\left(\mathbf{h}_{n}, \mathbf{x}_{n} \mid \mathbf{h}_{0: n-1}, \mathbf{x}_{0: n-1}\right)
\end{aligned}
$$

But, unfortunately, attributed to the dynamic characteristics of the proposed DSM, the involved prior probability $p\left(\mathbf{h}_{n}, \mathbf{x}_{n} \mid \mathbf{h}_{0: n-1}, \mathbf{x}_{0: n}-1\right)$ and likelihood function $p\left(y_{n}{ }^{\dagger} \mid \mathbf{h}_{0: n}{ }^{\dagger}, \mathbf{x}_{0: n}{ }^{\dagger}, \mathbf{y}_{0: n}{ }^{\dagger}-1\right)$ are time-variant. As a result, the above sequential MAP procedure represented in (25) is only a theoretical solution of the Bayesian statistical inference, and it is almost impossible to derive in practical applications, so we should resort to a flexible method to approximate the complex distribution.

As a powerful methodology for sequential signal processing, PF could address this approximation problem effectively, and we will describe this technology in detail in the next part.

\subsection{PF technology}

$\mathrm{PF}$ is a sequential Monte Carlo methodology based on sequential importance sampling (SIS). The basic idea of PF is that the probability distribution of estimation is approximated with discrete random particles which are assigned to different weights, and it could be represented as (26)

$$
\hat{z}_{0: n}^{(\mathrm{MAP})}=\arg \max _{z_{0: n}} p\left(z_{0: n} \mid y_{0: n}\right) \approx \arg \max _{z_{0: n}}\left[\sum_{i=1}^{I} w_{n}^{(i)} \delta\left(z_{0: n}-z_{0: n}^{(i)}\right)\right]
$$

Here, $z(i) n$ represents the particles whose associated normalized weights are denoted by $w(i) n$, and $I$ represents the total number of particles. $\delta($.$) is the Dirac delta$ function, $\delta()=$.1 if $z=z^{(i)}$ and $\delta()=$.0 otherwise. The marginal data detection of (26) at time $n$ could be calculated by

$$
\hat{z}_{n}^{(\mathrm{MAP})}=\arg \max _{z_{n}}\left[\sum_{i=1}^{I} w_{n}^{(i)} \delta\left(z_{n}-z_{n}^{(i)}\right)\right]
$$

It could be obtained from (27) that the main operation steps of PF are generating particles and updating the associated weights when the new observation arrives. The formulas for computing are shown as (28), (29), and (30) [27].

$$
z_{n}^{(i)} \sim \pi\left(z_{n} \mid z_{0: n-1}, y_{0: n}\right)
$$

$$
\begin{aligned}
& \tilde{w}_{n}^{(i)}=w_{n-1}^{(i)} \frac{p\left(y_{n} \mid z_{0: n}^{(i)}, y_{0: n-1}\right)}{\pi\left(z_{n}^{(i)} \mid z_{0: n-1}^{(i)}, y_{0: n}\right)} \\
& w_{n}^{(i)}=\frac{\tilde{w}_{n}^{(i)}}{\sum_{i=0}^{I} \tilde{w}_{n}^{(i)}}
\end{aligned}
$$

Here, the $\pi\left(z_{n} \mid z_{0: n-1}, y_{0: n}\right)$ denotes the importance PDF with the same support of PDF $p\left(z_{n} \mid z_{0: n-1}, y_{0: n}\right)$ but is much easier to sample from. It should be noted that the importance PDF and the likelihood function $p\left(y_{n} \mid z(i) 0\right.$ : $\left.n-1, y_{0: n-1}\right)$ are not fixed but changes according to practical applications. To sum up, the concrete procedure of basic PF algorithm is presented in Algorithm 1 [27].

Algorithm 1 PF algorithm for MAP estimation

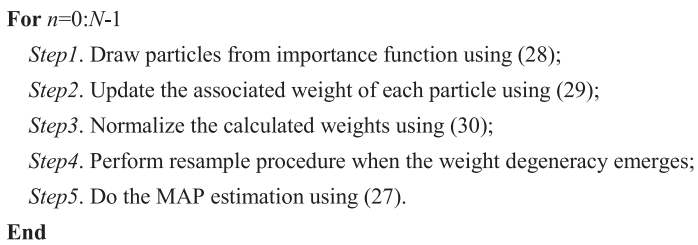

\section{Joint estimation algorithm}

The fading channel considered is set to be slow fading, and the coherence time of channel $T \mathrm{c}$ is multiples of $T \mathrm{~s}$, i.e., $T \mathrm{c}=J T \mathrm{~s}$. It means that the channel gain remains constant in the $j \times J$ th to $(j+1) \times J-1$ th sensing slot, here, 
$j=0,1 . ., N / J$. In order to improve the accuracy of estimation, the channel gain will be estimated in every sensing slot based on the real-time observation. But, unfortunately, it is apparent from (20) that the channel gain $\mathbf{h}_{n}$ will disappear thoroughly when the $\mathrm{PU}$ is inactive, i.e., $\mathbf{x}_{n}=\mathbf{0}$, so the estimation of the channel gain becomes unconquerable in this situation. Besides, the channel gain is possible to transfer into another state only in the first slot. For reasons as noted above, we design different mechanisms to estimate the channel gain which is more practical and flexible. More specifically, the novel estimation algorithm designed consists of three steps. i.e., (1) coarse detection, where the PU state is detected roughly in this step; (2) the estimation of fading channel gain based on the MAP criterion; and (3) the PF-based PU state detection. These steps will be described in detail as follows.

\subsection{Coarse detection}

The purpose of this step is to detect the PU state roughly in order to provide a reference for the mechanism choice of channel gain. We get an initial result by comparing the observation with a specified threshold; the threshold is achieved by

$$
\tau_{\mathrm{CD}}=\frac{1}{2} \times\left[\left(\mathbf{s}_{\mathrm{c}} \otimes \mathbf{h}_{\mathrm{min}}\right) \otimes \mathbf{s}_{\mathrm{c}}\right]
$$

Here, $\mathbf{h}_{\min }$ represents a vector which consists of the minimum amplitude of every channel, $\mathbf{h}_{\min }=\left[\min \left(h_{0,0: K}-1\right)\right.$, $\left.\min \left(h_{1,0: K}-1\right), \ldots, \min \left(h_{L}-1,0: K-1\right)\right]^{T}$. Based on the decision criterion above, we could get the coarse detection as

$$
\mathbf{x}_{n}^{\dagger}= \begin{cases}\mathbf{0} & y_{n}<\tau_{\mathrm{CD}} \\ \mathbf{s}_{\mathrm{c}} & y_{n} \geq \tau_{\mathrm{CD}}\end{cases}
$$

Note that the purpose of coarse detection in this investigation is to provide the basis for the choice of mechanism which will be applied to estimate the channel gain. Even though the accuracy of coarse detection is relatively low, the subsequent step will modify the estimation result for the purpose of accurate detection.

\subsection{Estimation of TVMFF channel gain}

In this section, we utilize three different mechanisms for estimating the TVMFF channel gain. The reasons for the choice are twofold. Firstly, as mentioned previously, the sensing slots are classified into two categories, and the channel gain is possible to transfer into another state only in the first slot. Secondly, the MAP criterion for estimating channel gain becomes useless when the $\mathrm{PU}$ state is inactive, i.e., $\mathbf{x}_{n}=\mathbf{0}$; therefore, we should resort to some other effective ways to combat this problem. Based on the two reasons mentioned previously, this paper presents the overall design diagram for the TVMFF channel, and it could be divided into three situations.

If $\mathbf{x}_{\mathbf{n}}^{\dagger}=\mathbf{0}$, and the sensing slot is the first in a channel state period, we could obtain the estimation of $\mathbf{h}_{n}$ only based on the prior transition probability of time-variant channel, as shown in (33).

$$
\hat{\mathbf{h}}_{n}=\arg \max _{\mathbf{h}_{n} \in \mathrm{H}} p\left(\mathbf{h}_{n} \mid \hat{\mathbf{h}}_{n-1}\right) \quad \mathbf{x}_{n}^{\dagger}=\mathbf{0} \text { \& first slot }
$$

If $\mathbf{x}_{\mathbf{n}}^{\dagger}=\mathbf{0}$, and the sensing slot is the non-first, the estimation of fading channel gain is assumed unchanged, like (34).

$$
\hat{\mathbf{h}}_{n}=\hat{\mathbf{h}}_{n-1}, \quad \mathbf{x}_{n}^{\dagger}=\mathbf{0} \text { and non-first slot }
$$

If $\mathbf{x}_{\mathbf{n}}^{\dagger}=\mathbf{s}_{\mathrm{c}}$, we could obtain the MAP estimation of channel gain by (35).

$$
\hat{\mathbf{h}}_{n}=\arg \max _{\mathbf{h}_{n} \in \mathrm{H}} p\left(\mathbf{h}_{n} \mid \hat{\mathbf{h}}_{0: n-1}, \hat{\mathbf{x}}_{0: n-1}, \mathbf{x}_{n}^{\dagger}=\mathbf{s}_{\mathrm{c}}, y_{0: n}\right)
$$

The posterior probability in (35) could be derived as (36), and a more detailed explanation of (36) is specified in Appendix 1.

$$
p\left(\mathbf{h}_{n} \mid \hat{\mathbf{h}}_{0: n-1}, \hat{\mathbf{x}}_{0: n-1}, \mathbf{x}_{n}^{\dagger}=\mathbf{s}_{\mathrm{c}}, y_{0: n}\right) \propto p\left(y_{n} \mid \mathbf{h}_{n}, \mathbf{x}_{n}^{\dagger}=\mathbf{s}_{\mathrm{c}}\right) p\left(\mathbf{h}_{n} \mid \hat{\mathbf{h}}_{n-1}\right)
$$

The likelihood function $p\left(y_{n} \mid \mathbf{h}_{n}, \mathbf{x} \dagger \mathbf{n}=\mathbf{s}_{\mathrm{c}}\right)$ in (36) follows the Gaussian distribution, and the configuration could be calculated by

$$
\begin{aligned}
p\left(y_{n} \mid \mathbf{h}_{n}, \mathbf{x}_{n}^{\dagger}=\mathbf{s}_{\mathrm{c}}\right)= & \frac{1}{\sqrt{2 \pi\left(\mathbf{s}_{\mathrm{c}}^{T} \times \mathbf{s}_{\mathrm{c}}\right) \sigma^{2}}} \\
& \exp \left\{\frac{-\left[y_{n}-\left(\mathbf{h}_{n} \otimes \mathbf{s}_{\mathrm{c}}\right) \otimes \mathbf{s}_{\mathrm{c}}\right]^{2}}{2\left(\mathbf{s}_{\mathrm{c}}^{T} \times \mathbf{s}_{\mathrm{c}}\right) \sigma^{2}}\right\}
\end{aligned}
$$

\subsection{PF-based PU state detection}

Based on the use of the Bayesian theory and SIS, particle filtering technology shows huge potential when handing 
with blind estimation problem. The key of particle filtering is approximating a complex distribution by a series of particles with associated weights and then the MAP estimation could be drawn, and the marginal data detection at time $n$ is given by [28]

$$
\hat{\mathbf{x}}_{n}^{(\mathrm{MAP})}=\arg \max _{\mathbf{x}_{n} \in \mathbf{X}}\left\{\sum_{i=1}^{I} \delta\left(\mathbf{x}_{n}-\mathbf{x}_{n}^{(i)}\right) w_{n}^{(i)}\right\}
$$

The process of PF technology applying for signal detection covers five basic steps as mentioned in Section 3.2. We will give a specific implementation program of these steps.

Step 1: We begin the SIS algorithm by drawing particles from the important probability distribution function. The form of the important function varies according to the actual situation. In this paper, we adopt the optimal importance function, and it could be written as

$$
\begin{aligned}
& \pi\left(\mathbf{x}_{n} \mid \mathbf{x}_{0: n-1}^{(i)}, \hat{\mathbf{h}}_{0: n}, y_{0: n}\right)=p\left(y_{n} \mid \mathbf{x}_{n}, \mathbf{x}_{0: n-1}^{(i)}, \hat{\mathbf{h}}_{0: n}, y_{0: n-1}\right) \\
& p\left(\mathbf{x}_{n} \mid \mathbf{x}_{0: n-1}^{(i)}, \hat{\mathbf{h}}_{0: n}, y_{0: n-1}\right) \approx p\left(y_{n} \mid \mathbf{x}_{n}, \hat{\mathbf{h}}_{n}\right) p\left(\mathbf{x}_{n} \mid \mathbf{x}_{n-1}^{(i)}\right)
\end{aligned}
$$

Step 2: The associated importance weights could be computed recursively by

$$
\tilde{w}_{n}^{(i)} \propto w_{n-1}^{(i)} \times \frac{p\left(y_{n} \mid \mathbf{x}_{0: n}^{(i)}, \hat{\mathbf{h}}_{n}, y_{0: n-1}\right)}{\pi\left(\mathbf{x}_{n}^{(i)} \mid \mathbf{x}_{0: n-1}^{(i)}, \hat{\mathbf{h}}_{n}, y_{0: n}\right)} \propto w_{n-1}^{(i)} \times p\left(y_{n} \mid \mathbf{x}_{n-1}^{(i)}, \hat{\mathbf{h}}_{n}\right)
$$

The likelihood function in (40) could be calculated by (41), that is,

$$
\begin{aligned}
p\left(y_{n} \mid x_{n-1}^{(i)}, \hat{\mathbf{h}}_{n}\right) & =\sum_{\mathbf{x}_{n}^{(i)} \in\left\{\mathbf{0}, \mathbf{s}_{c}\right\}} p\left(y_{n} \mid \mathbf{x}_{n}^{(i)}, \mathbf{x}_{n-1}^{(i)}, \hat{\mathbf{h}}_{n}\right) p\left(\mathbf{x}_{n}^{(i)} \mid \mathbf{x}_{n-1}^{(i)}\right) \\
& =\sum_{x_{n}^{(i)} \in\left\{\mathbf{0}, \mathbf{s}_{\mathbf{c}}\right\}} p\left(y_{n} \mid \mathbf{x}_{n}^{(i)}, \hat{\mathbf{h}}_{n}\right) p\left(\mathbf{x}_{n}^{(i)} \mid \mathbf{x}_{n-1}^{(i)}\right)
\end{aligned}
$$

The likelihood function in (41) could be calculated by (42) and (37):

$$
\begin{aligned}
p\left(y_{n} \mid \mathbf{x}_{n}^{\dagger}=\mathbf{0}, \hat{\mathbf{h}}_{n}\right)= & \frac{1}{\sqrt{2 \pi\left(\mathbf{s}_{\mathrm{c}}^{T} \times \mathbf{s}_{\mathrm{c}}\right) \sigma^{2}}} \\
& \exp \left\{\frac{-\left[y_{n}\right]^{2}}{2\left(\mathbf{s}_{\mathrm{c}}^{T} \times \mathbf{s}_{\mathrm{c}}\right) \sigma^{2}}\right\}
\end{aligned}
$$

Step 3: Normalize the calculated importance weights using (30).

Step 4: The degeneracy of weight is usually inevitable after several times of iteration, and weight degeneracy may cause the approximation of posterior probability to deteriorate seriously and even become useless. Resampling procedure is proposed to alleviate this problem efficiently. The main idea of re-sampling is to eliminate particle trajectories with small normalized importance weight while concentrating upon those particles which have larger normalized importance weight, and implementation procedures are shown as follows [27]:

Set $N_{\text {eff }}=\frac{1}{\sum_{i=1}^{I}\left[w_{n}^{(i)}\right]^{2}}$ and choose the value of $\varepsilon$ with the limitation $0<\varepsilon<1$.

If $N_{\text {eff }}<\varepsilon$, then.

For $i=1: I$, set $\tilde{\mathbf{x}}_{0: n}^{(i)}=\mathbf{x}_{0: n}^{(i)}$ with weight $w_{n}^{(i)}$;

Set $\mathbf{x}_{0: n}^{(i)}=\tilde{\mathbf{x}}_{0: n}^{(i)}$ and $w_{n}^{(i)}=1 / I, i=1,2, \ldots, I$.

Step 5: The MAP estimation could be achieved by (38) based on the particles and associated weights computed above.

\subsection{Implement}

Based on the elaborations above, the joint detection algorithm comes in its fullness. At the receiver end, we could detect the PU state in the following three steps.

\footnotetext{
For $n=0: N-1$

Step1. By comparing the current observation $y_{n}$ with the threshold $\tau_{C D}$, the result of coarse detection is produced;

Step2. Based on the result of coarse detection and the location of sensing slot in a channel gain period, the estimation mechanism of fading channel state is chosen and performed;

Step3. Relying on the observation and the estimation of fading channel gain, the PU state could be estimated by utilizing PF technique.

End
} 


\section{Simulation experiments}

In this investigation, the false alarm $p_{\mathrm{f}}$ and the missing alarm $p_{\mathrm{m}}$ will be considered in a comprehensive way. In other words, we adopt a novel definition of detection probability, and it could be written as

$$
\begin{aligned}
p_{d} & \triangleq p\left(\hat{x}_{n}=\mathbf{0} \mid H_{0}\right) p\left(H_{0}\right)+p\left(\hat{x}_{n}=\mathbf{s}_{c} \mid H_{1}\right) p\left(H_{1}\right) \\
& =1-p_{f} p\left(H_{0}\right)-p_{m} p\left(H_{1}\right)
\end{aligned}
$$

In this investigation, we firstly compare the sensing performance of traditional MFD, proposed algorithm, and CAV detection algorithm which is newly introduced and has found a wide application recently. Secondly, we mainly study the effects of the following three parameters of wireless communication environment have on the sensing performance, i.e., Doppler frequency shift $f_{\mathrm{d}}$, number of multipath channel $L$, and sampling size in one sensing slot $M$. In addition, we will evaluate the MSE performance of the unknown multipath channel.

\subsection{Comparison of sensing performance}

In this simulation, the number of multipath $L$ is set to be 4 , the size of partitioned channel states number $K$ is set to be 5 , the mean of multipath taps is configured to $\mathrm{E}\{\mathbf{h}\}=[1,0.1,0.02,0.01]$, and the sampling size $M=7$. It is obvious from Figure 5 that compared with the two other methods, the sensing performance achieved by the proposed algorithm could be improved significantly. For example, when the detection probability surpass 0.95 and the $f_{\mathrm{d}}=0.1$, the desired SNR of the new algorithm and traditional MFD method is 4.5 and $12 \mathrm{~dB}$, respectively.
Furthermore, it should be noted that the performance of the CAV detection is unacceptable. That is because the validity of the CAV algorithm relies on the assumption that the sampled signals are correlated, and the correlation of the received signals in the model proposed is lower.

\subsection{Doppler frequency shift}

In this simulation, the number of multipath $L$ is set to be 4, the size of partitioned channel states number $K$ is set to be 5 , the mean of multipath taps is configured to $\mathrm{E}\{\mathbf{h}\}=[1,0.1,0.02,0.01]$, and the sampling size $M=7$. Three configurations of maximum Doppler frequency shift $f_{\mathrm{d}}$ are adopted in this experiment, i.e., $f_{\mathrm{d}}=0.2,0.1$, 0.05 . The simulation results are shown in Figure 6.

It is obvious from Figure 6 that compared with the traditional MFD method, the sensing performance achieved by the proposed algorithm could be improved significantly when operating at the TVMFF channel. In addition, from Figure 6, it is seen in a clear logical way that the performance of the proposed method will degrade as the value of the Doppler spread increases.

\subsection{Number of multipath channel}

In this simulation, the maximum Doppler frequency shift $f_{\mathrm{d}}=0.1, K=5$, and the sampling size $M=7$. The number of multipath channel $L$ is 3,4 , and 5 , respectively. The average values of the multipath taps are configured to $\mathrm{E}\{\mathbf{h}\}=[1,0.1,0.02], \mathrm{E}\{\mathbf{h}\}=[1,0.1,0.02,0.01]$, and $E\{\mathbf{h}\}=[1,0.1,0.02,0.01,0.005]$. From the numerical experiment results shown in Figure 7, it should be noted that with the advance of $L$, both the sensing performance

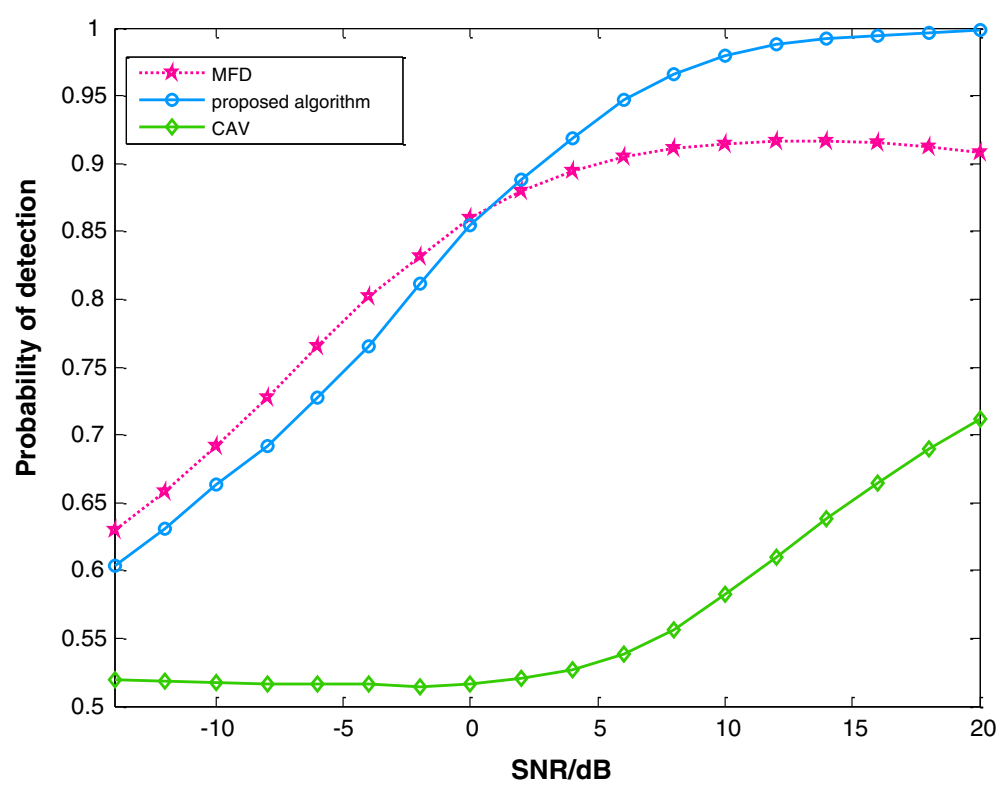

Figure 5 Comparison of sensing performance achieved by traditional MFD, CAV detection, and the proposed algorithm. 


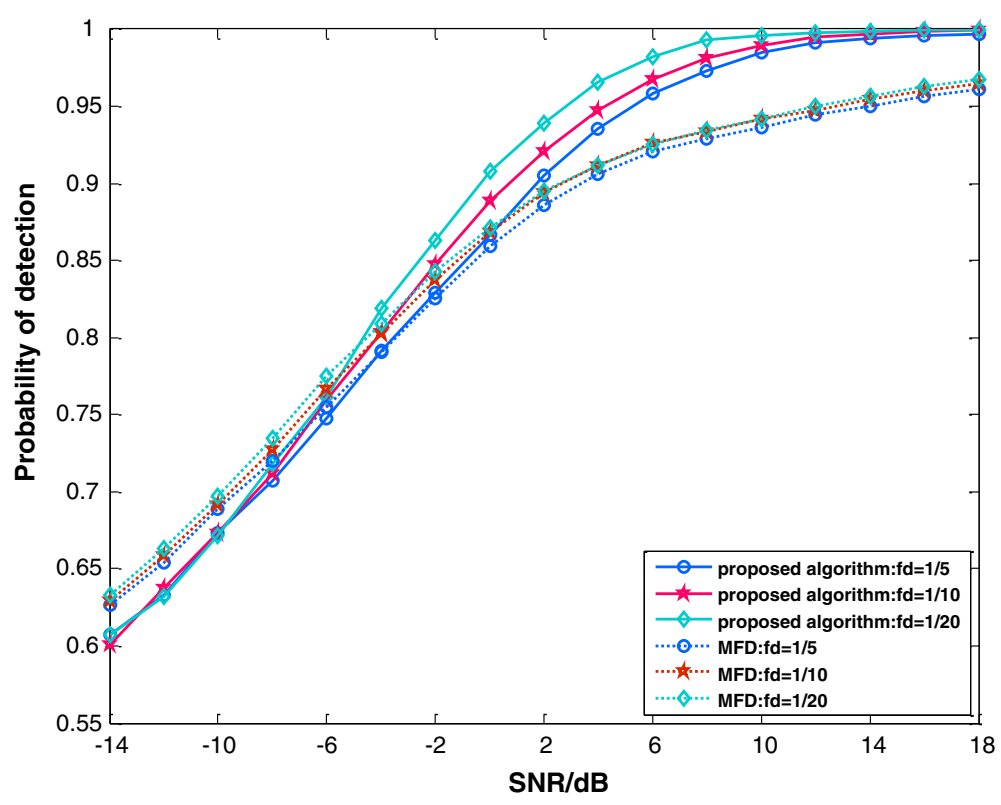

Figure 6 Sensing performance under different maximum Doppler frequency shifts $f_{\mathrm{d}}$.

achieved by the proposed algorithm and the traditional MFD will decrease. That is because the greater the number of channel, the more sensing performance suffers from intersymbol interference (ISI).

\subsection{Sampling size}

In this simulation, $f_{\mathrm{d}}=0.1, K=5, L=4$, and $\mathrm{E}\{\mathbf{h}\}=$ $[1,0.1,0.02,0.01]$. The configurations of the sampling size $M$ are set to be 7,15 , and 31 . From the numerical experiment results shown in Figure 8 , it should be noted that with the advance of $M$, the performance of proposed algorithm will increase obviously. That is because the greater the number of sampling size, the more information of PU that SU could get from the observations. As a result, the accuracy of the estimation increases.

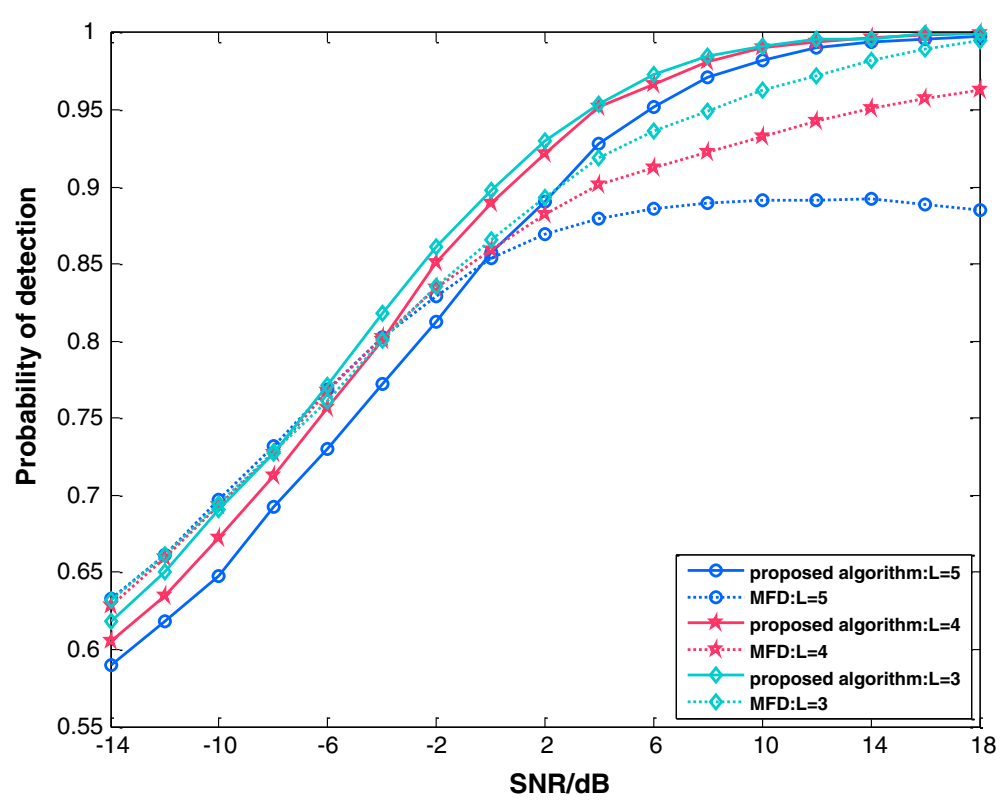

Figure 7 Sensing performance under different numbers of multipath $L$. 


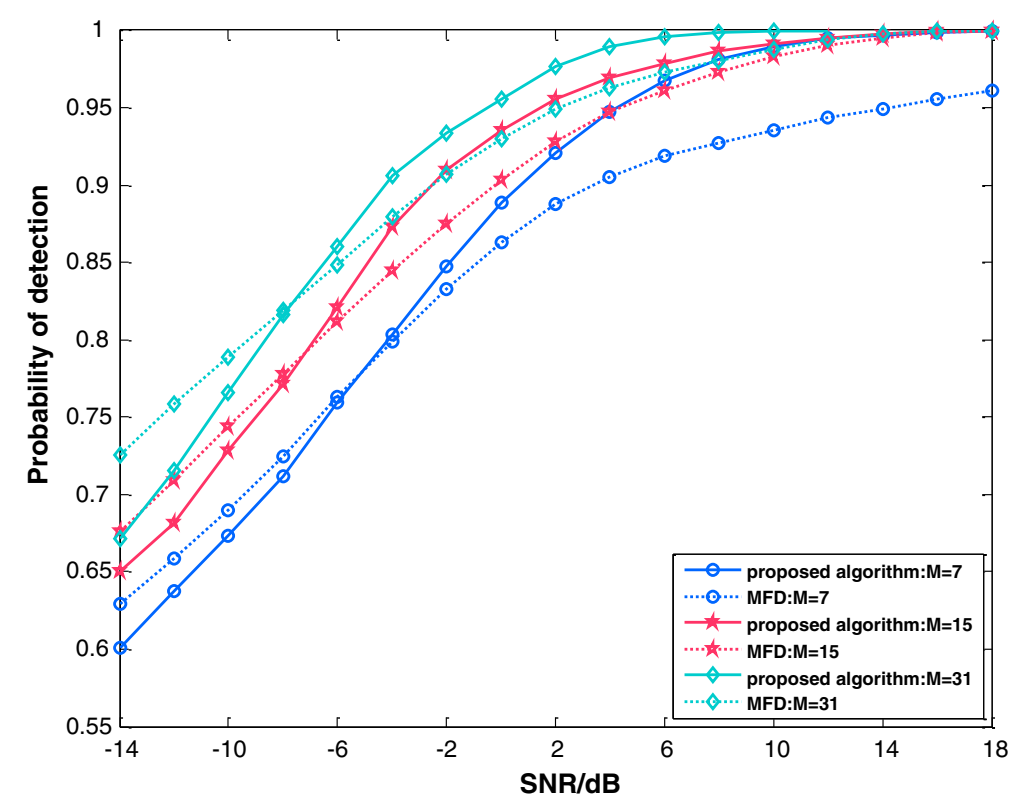

Figure 8 Sensing performance under different sampling sizes $M$.

\subsection{MSE of estimations}

The purpose of this experiment is to evaluate the MSE performance of the multipath channel estimated. The MSE is defined as

$$
\mathrm{MSE}_{\hat{\mathbf{h}}}=\mathrm{E}\left[\frac{1}{N} \sum_{n=0}^{N}\left\|\hat{\mathbf{h}}_{n}-\mathbf{h}_{n}\right\|^{2}\right]
$$

In this simulation, $f_{\mathrm{d}}=0.1, K=5, M=15$. The configurations of multipath channel $L$ are 3,4 , and 5, and the average values of multipath taps are configured to $\mathrm{E}\{\mathbf{h}\}=[1,0.1,0.02], \mathrm{E}\{\mathbf{h}\}=[1,0.1,0.02,0.01]$, and $\mathrm{E}\{\mathbf{h}\}=$ $[1,0.1,0.02,0.01,0.005]$. It has been demonstrated in Figure 9 that with the improvement of $L$, the MSE will decrease and the estimation result of channel gain achieved by the proposed algorithm is comparatively accurate.

\section{Conclusion}

In order to troubleshoot the problem in practical spectrum sensing results from multipath time-variant fading channels,

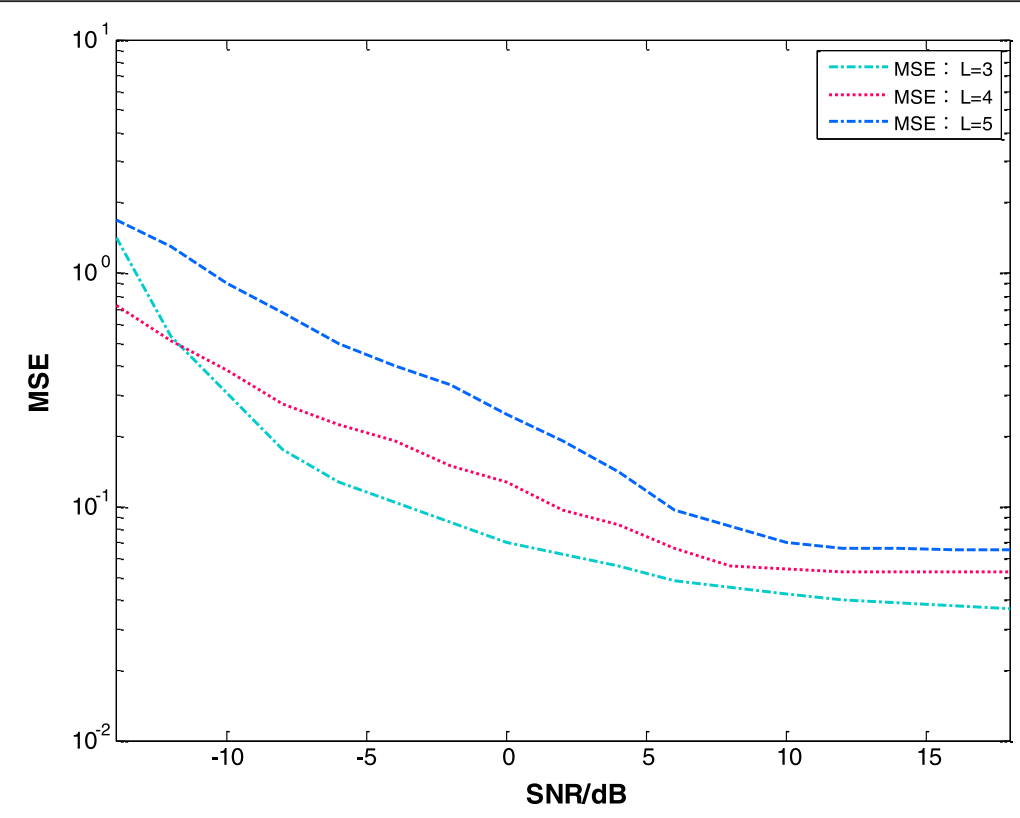

Figure 9 MSE performance under different numbers of multipath $L$. 
a promising spectrum sensing algorithm which could find wide use in CR systems is proposed in this article. The advantages of this algorithm are fivefold. First of all, TVMFF channels are common in the next-generation communication systems such as mobile communication and indoor communication. It is a grim challenge to perform spectrum sensing under TVMFF channel. The algorithm designed could overcome this shortcoming effectively. Secondly, because the spectrum sensing could be achieved by detecting whether the pilot or synchronization code exists, the algorithm proposed could be applied for most CR systems, and it inherits the advantages of MFD such as rapidity and accuracy. Thirdly, a novel DSM is established by making a liberal allowance for the dynamic evolution behaviors of the TVMFF channel and PU state. This DSM could be applied extensively for signal estimation in other communication systems. Fourthly, by resorting to MAP criterion and PF methodology, we propose a joint estimation algorithm which could estimate the channel gain and PU state sequentially and timely without a training sequence, and simulation experiments indicate that this method could achieve better performance than the other methods over TVMFF channel. Finally, the MAP criterion is easy to accomplish in practice, and the number of particles is in small batches or singly; therefore, the complexity of this algorithm could be controlled to manageable proportions, and the feasibility could be ensured. Because of the aforementioned advantages, this proposed method could provide a referential solution to the practical design of spectrum sensing algorithms and improve the sensing performance in practical CR networks significantly.

\section{Appendix 1}

Posterior probability of TVMFF channel gain

The posterior probability of $\mathbf{h}_{n}$ could be represented as Equation 45:

$$
p\left(\mathbf{h}_{n} \mid \hat{\mathbf{h}}_{0: n-1}, \hat{\mathbf{x}}_{0: n-1}, \mathbf{x}_{n}^{\dagger}=\mathbf{s}_{\mathrm{c}}, y_{0: n}\right)
$$

Based on the conditional probability $p(\mathrm{~B} \mid \mathrm{A})=p(\mathrm{AB}) / p$ (A), we could rewrite Equation 45 as
It is obvious from Equation (20) that the observation $y_{n}$ is only associated with the channel gain and PU state in current sensing slot; thus, the simplification could be performed as follows:

$$
\begin{aligned}
& p\left(y_{n} \mid \mathbf{h}_{n}, \hat{\mathbf{h}}_{0: n-1}, \hat{\mathbf{x}}_{0: n-1}, \mathbf{x}_{n}^{\dagger}=\mathbf{s}_{\mathrm{c}}, y_{0: n-1}\right)=p\left(y_{n} \mid \mathbf{h}_{n}, \mathbf{x}_{n}^{\dagger}=\mathbf{s}_{\mathrm{c}}\right) \\
& p\left(y_{n} \mid \hat{\mathbf{h}}_{0: n-1}, \hat{\mathbf{x}}_{0: n-1}, \mathbf{x}_{n}^{\dagger}=\mathbf{s}_{\mathrm{c}}, y_{0: n-1}\right)=p\left(y_{n} \mid \mathbf{x}_{n}^{\dagger}=\mathbf{s}_{\mathrm{c}}\right)
\end{aligned}
$$

Furthermore, the channel gain and the PU state are independent with each other; thus,

$$
p\left(\mathbf{h}_{n} \mid \hat{\mathbf{h}}_{0: n-1}, \hat{\mathbf{x}}_{0: n-1}, \mathbf{x}_{n}^{\dagger}=\mathbf{s}_{\mathrm{c}}, y_{0: n-1}\right)=p\left(\mathbf{h}_{n} \mid \hat{\mathbf{h}}_{0: n-1}\right)
$$

In combination, Equation 46 could be simplified as

$$
\begin{aligned}
& p\left(\mathbf{h}_{n} \mid \hat{\mathbf{h}}_{0: n-1}, \hat{\mathbf{x}}_{0: n-1}, \mathbf{x}_{n}^{\dagger}=\mathbf{s}_{\mathrm{c}}, y_{0: n}\right) \\
= & \frac{p\left(y_{n} \mid \mathbf{h}_{n}, \mathbf{x}_{n}^{\dagger}=\mathbf{s}_{\mathrm{c}}\right) p\left(\mathbf{h}_{n} \mid \hat{\mathbf{h}}_{0: n-1}\right)}{p\left(y_{n} \mid \mathbf{x}_{n}^{\dagger}=\mathbf{s}_{\mathrm{c}}\right)} \\
\propto & p\left(y_{n} \mid \mathbf{h}_{n}, \mathbf{x}_{n}^{\dagger}=\mathbf{s}_{\mathrm{c}}\right) p\left(\mathbf{h}_{n} \mid \hat{\mathbf{h}}_{0: n-1}\right)
\end{aligned}
$$

We have declared that the first-order FSMC is adopted in our investigation to model the TVMFF channel, so the current channel state is only associated with the right prior state while irrelevant to all other past or future channel states. For this reason, a final conclusion has been arrived:

$$
p\left(\mathbf{h}_{n} \mid \hat{\mathbf{h}}_{0: n-1}, \hat{\mathbf{x}}_{0: n-1}, \mathbf{x}_{n}^{\dagger}=\mathbf{s}_{\mathrm{c}}, y_{0: n}\right) \propto p\left(y_{n} \mid \mathbf{h}_{n}, \mathbf{x}_{n}^{\dagger}=\mathbf{s}_{\mathrm{c}}\right) p\left(\mathbf{h}_{n} \mid \hat{\mathbf{h}}_{n-1}\right)
$$

$$
\begin{aligned}
& p\left(\mathbf{h}_{n} \mid \hat{\mathbf{h}}_{0: n-1}, \hat{\mathbf{x}}_{0: n-1}, \mathbf{x}_{n}^{\dagger}=\mathbf{s}_{\mathrm{c}}, y_{0: n}\right)=\frac{p\left(\mathbf{h}_{n}, \hat{\mathbf{h}}_{0: n-1}, \hat{\mathbf{x}}_{0: n-1}, \mathbf{x}_{n}^{\dagger}=\mathbf{s}_{\mathrm{c}}, y_{0: n}\right)}{p\left(\hat{\mathbf{h}}_{0: n-1}, \hat{\mathbf{x}}_{0: n-1}, \mathbf{x}_{n}^{\dagger}=\mathbf{s}_{\mathrm{c}}, y_{0: n}\right)} \\
= & \frac{p\left(y_{n} \mid \mathbf{h}_{n}, \hat{\mathbf{h}}_{0: n-1}, \hat{\mathbf{x}}_{0: n-1}, \mathbf{x}_{n}^{\dagger}=\mathbf{s}_{\mathrm{c}}, y_{0: n-1}\right) p\left(\mathbf{h}_{n}, \hat{\mathbf{h}}_{0: n-1}, \hat{\mathbf{x}}_{0: n-1}, \mathbf{x}_{n}^{\dagger}=\mathbf{s}_{\mathrm{c}}, y_{0: n-1}\right)}{p\left(\hat{\mathbf{h}}_{0: n-1}, \hat{\mathbf{x}}_{0: n-1}, \mathbf{x}_{n}^{\dagger}=\mathbf{s}_{\mathrm{c}}, y_{0: n}\right)} \\
= & \frac{p\left(y_{n} \mid \mathbf{h}_{n}, \hat{\mathbf{h}}_{0: n-1}, \hat{\mathbf{x}}_{0: n-1}, \mathbf{x}_{n}^{\dagger}=\mathbf{s}_{\mathrm{c}}, y_{0: n-1}\right) p\left(\mathbf{h}_{n} \mid \hat{\mathbf{h}}_{0: n-1}, \hat{\mathbf{x}}_{0: n-1}, \mathbf{x}_{n}^{\dagger}=\mathbf{s}_{\mathrm{c}}, y_{0: n-1}\right) p\left(\hat{\mathbf{h}}_{0: n-1}, \hat{\mathbf{x}}_{0: n-1}, \mathbf{x}_{n}^{\dagger}=\mathbf{s}_{\mathrm{c}}, y_{0: n-1}\right)}{p\left(y_{n} \mid \hat{\mathbf{h}}_{0: n-1}, \hat{\mathbf{x}}_{0: n-1}, \mathbf{x}_{n}^{\dagger}=\mathbf{s}_{\mathrm{c}}, y_{0: n-1}\right) p\left(\hat{\mathbf{h}}_{0: n-1}, \hat{\mathbf{x}}_{0: n-1}, \mathbf{x}_{n}^{\dagger}=\mathbf{s}_{\mathrm{c}}, y_{0: n-1}\right)} \\
= & \frac{p\left(y_{n} \mid \mathbf{h}_{n}, \hat{\mathbf{h}}_{0: n-1}, \hat{\mathbf{x}}_{0: n-1}, \mathbf{x}_{n}^{\dagger}=\mathbf{s}_{\mathrm{c}}, y_{0: n-1}\right) p\left(\mathbf{h}_{n} \mid \hat{\mathbf{h}}_{0: n-1}, \hat{\mathbf{x}}_{0: n-1}, \mathbf{x}_{n}^{\dagger}=\mathbf{s}_{\mathrm{c}}, y_{0: n-1}\right)}{p\left(y_{n} \mid \hat{\mathbf{h}}_{0: n-1}, \hat{\mathbf{x}}_{0: n-1}, \mathbf{x}_{n}^{\dagger}=\mathbf{s}_{\mathrm{c}}, y_{0: n-1}\right)}
\end{aligned}
$$




\section{Competing interests}

The authors declare that they have no competing interest.

\section{Acknowledgements}

This work was supported by the National Natural Science Foundation of China $(61271180,61379016)$, Fundamental Research Funds for the Central Universities (2014RC0101), Major National Science and Technology Projects (2013ZX03001015-003), Postgraduate Innovation Fund of SICE, BUPT 2013, and Research Fund for the Doctoral Program of Higher Education of China (20130005110016)

\section{Author details}

'Beijing University of Posts and Telecommunications (BUPT), Haidian, Beijing 100876, China. ${ }^{2}$ The State Radio Monitoring Center Testing Center, Xicheng District, Beijing 100041, China.

Received: 20 December 2013 Accepted: 5 May 2014

Published: 22 May 2014

\section{References}

1. Federal Communication Commission, Spectrum Policy Task Force Report. ET Docket, no. 02-155, Nov 02, 2002 (FCC, Washington DC, 2002)

2. J Mitola, GQ Maguire, Cognitive radio: making software radios more personal. IEEE Personal Commun. 6(4), 13-18 (1999)

3. H Simon, Cognitive radio: brain-empowered wireless communication. IEEE J. Selected Areas Commun. 23(2), 201-220 (2005)

4. B Wang, KJR Liu, Advances in cognitive radio networks: a survey. IEEE J. Selected Topics Signal. Process. 5, 5-23 (2011)

5. M Jun, G Li, Y Biing, H Juang, Signal processing in cognitive radio. Proc. IEEE 97(5), 805-823 (2009)

6. CR Stevenson, C Cordeiro, E Sofer, G Chouinard, Functional Requirements for the 802.22 WRAN Standard: IEEE 802.22-05/0007r46 (IEEE, Piscataway, 2005)

7. IEEE, Draft Supplement to Standard for Telecommunications and Information Exchange Between Systems - LAN/MAN Specific Requirements - Part 11 (2003) Wireless Medium Access Control (MAC) and Physical Layer (PHY) Specifications: Specification for Radio Resource Measurement: IEEE 802.11 k/D0.7 (IEEE, Piscataway, 2003)

8. Bluetooth Special Interest Group, Specification of the Bluetooth System, Master Table of Contents \& Compliance Requirements (SIG, Kirkland, 2004)

9. T Yucek, H Arslan, A survey of spectrum sensing algorithms for cognitive radio applications. IEEE Commun. Surv. Tutorials 11(1), 116-130 (2009)

10. M López-Benítez, F Casadevall, Improved energy detection spectrum sensing for cognitive radio. IET Commun. 6(8), 785-796 (2012)

11. FF Digham, MS Alouini, MK Simon, Energy detection of unknown signals over fading channels (Paper presented at the IEEE international conference on communications (ICC), Anchorage, AK, USA, 2003), pp. 3575-357

12. HS Chen, W Gao, DG Daut, Signature based spectrum sensing algorithms for IEEE 802.22 WRAN (Paper presented at the IEEE international conference on communications (ICC), Glasgow, Scotland, 2007), pp. 6487-6492

13. Z Zhang, QQ Yang, LK Wang, XF Zhou, A novel hybrid matched Filter structure for IEEE 802.22 standard (Paper presented at the IEEE Asia Pacific conference on circuits and systems (APCCAS), Kuala Lumpur, Malaysia, 2010), pp. 652-655

14. SL Sabat, S Srinu, A Raveendranadh, SK Udgata, Spectrum sensing based on entropy estimation using cyclostationary features for cognitive radio (Paper presented at the fourth international conference on communication systems and networks (COMSNETS), Bangalore, India, 2012), pp. 1-6

15. H Tang, Some physical layer issues of wide-band cognitive radio systems (Paper presented at the IEEE international symposium on new frontiers in dynamic spectrum access networks. Baltimore, MD, USA, 2005), pp. 151-159

16. R Tandra, A Sahai, Fundamental limits on detection in low SNR under noise uncertainty, vol. 1 (Paper presented at the international conference on wireless networks, communications and mobile computing, Maui, HI, USA, 2005), pp. 464-469

17. P Sadeghi, RA Kennedy, PB Rapajic, R Shams, Finite-state Markov modeling of fading channels. IEEE Signal Process. Mag. 25(5), 57-80 (2008)

18. Z Yonghong, L Ying-Chang, Spectrum-sensing algorithms for cognitive radio based on statistical covariances. IEEE Trans. Vehicular Technol. 58(4), 1804-1815 (2009)

19. PM Djuric, JH Kotecha, JQ Zhang, YF Huang, T Chirmai, MF Bugallo, J Miguez, Particle filtering. IEEE Signal Processing Mag. 20(5), 19-38 (2003)
20. D Cabric, A Tkachenko, RW Brodersen, Spectrum sensing measurements of pilot, energy, and collaborative detection (Paper presented at the IEEE military communications conference (MILCOM), Washington D.C., USA 2006), pp. 1-7

21. J Won Gi, C Kyung Hi, C Yong Soo, An equalization technique for orthogonal frequency-division multiplexing systems in time-variant multipath channels. IEEE Trans. Commun. 47(1), 27-32 (1999)

22. P Sadeghi, RA Kennedy, PB Rapajic, R Shams, Finite-state Markov modeling of fading channels: a survey of principles and applications. IEEE Signal Process. Mag. 25(5), 57-80 (2008)

23. PO Vontobel, A Kavcřć, DM Arnold, HA Loeliger, A generalization of the Blahut-Arimoto algorithm to finite-state channels. IEEE Trans. Inf. Theory 54(5), 1887-1918 (2008)

24. JG Proakis, Digital Communications, 4th edn. (McGraw-Hill, New York, 2002)

25. V Nithya, $\vee$ Bhaskar, Finite-state Markov channel modelling under jointly varying amplitude and phase in time-varying flat-fading channels. IET Commun. 5(9), 1237-1245 (2011)

26. HS Wang, N Moayeri, Finite-state Markov channel-A useful model for radio communication channels. IEEE Trans. Veh. Technol. 44(1), 163-171 (1995)

27. J Miguez, PM Djuric, Blind equalization by sequential importance sampling, 1st edn. (Paper presented at the IEEE international symposium on circuits and systems (ISCAS), Scottsdale, AR, USA, 2002), pp. I-845-I-848

28. M Joaquin, PM Djuric, Blind equalization of frequency-selective channels by sequential importance sampling. IEEE Trans. Signal Process. 52(10), 2738-2748 (2004)

\section{doi:10.1186/1687-1499-2014-84}

Cite this article as: Zhao et al.: Blind spectrum sensing for cognitive radio over time-variant multipath flat-fading channels. EURASIP Journal on Wireless Communications and Networking 2014 2014:84.

\section{Submit your manuscript to a SpringerOpen ${ }^{\odot}$ journal and benefit from:}

- Convenient online submission

- Rigorous peer review

- Immediate publication on acceptance

- Open access: articles freely available online

- High visibility within the field

- Retaining the copyright to your article

Submit your next manuscript at $>$ springeropen.com 